\title{
Determinants of stress for staff in a neonatal intensive care unit
}

\author{
J ASTBURY AND V Y H YU \\ Department of Paediatrics, Queen Victoria Medical Centre, Melbourne, Australia
}

SUMMARY Components of stress for 22 paediatric consultants and 29 nursing sisters working in neonatal intensive care units were studied. Ten situations ${ }^{1}$ were rated for their intensity of stress and their frequency of occurrence. For intensity of stress, significantly more paediatric consultants rated the competing demands of personal life versus work as highly stressful, than did nursing sisters. For frequency of stress, significantly more paediatric consultants rated 4 situations as frequently occurring than did nursing sisters (priorities of care, understaffing/overwork, personal life versus work, condition of outborn infants). However, significantly more nursing sisters found problems in working with their peers a frequently occurring stress than paediatric consultants. Total scores calculated for intensity and frequency of stress showed that although the overall intensity of stress experienced was similar, paediatric consultants had a significantly higher mean score for frequency of stress than nursing sisters in the neonatal intensive care unit.

During the last 10 years, several reports have appeared in the medical and behavioural science literature on the psychological impact of working in a neonatal intensive care unit (NICU). ${ }^{2-4}$ The cumulative stress derived from working in an NICU has even been given a name, 'burnout', by Marshall and $\mathrm{Kasman}^{5}$ who define it as 'the loss of motivation for creative involvement'.

While there has been consensus that the environment of the NICU is stressful, explanations of what constitutes that stress have been diverse. Stress has been attributed to the physical and emotional availability of staff to families 24 hours a day as a result of open visiting policies. It has been linked to the complex ethical dilemmas over prolongation of life made possible by a burgeoning technology. It has been said to be caused by the dual demands imposed by under-staffing and overwork, producing what one researcher called the 'dumped-on' syndrome. ${ }^{6}$ Other contributing factors mentioned have been the lack of role definition for NICU nurses and the resulting conflicts over decision-making and responsibility; the difficulty of keeping up with a constantly changing technology and medical body of knowledge concerning neonatal intensive care, when a failure to do so directly threatens self-esteem and perceived competence; and the stress produced by the physical environment of an NICU, with its distinctive noises, lights, sights, and smells. Overriding all these factors, the staff may experience a constant anticipation of crisis, so that 'quiet' times in the unit are not experienced as relaxing, but as uncomfortably empty and tense preludes to the next inevitable time of stress.

Studies undertaken so far into the stress associated with working in an NICU have concentrated on nursing staff and they have been descriptive and impressionistic, with an emphasis on clinically-based notions of coping with stress. The preponderance of descriptive studies over empirically based ones has left a number of questions unanswered. There exists the curious situation of coping strategies being recommended, in the absence of data on exactly what staff in an NICU find stressful or exactly which situations create coping difficulties. The present study was designed to answer the following questions. Is there a hierarchy of importance among factors cited as stressful ? Do different personnel-for example, nursing sisters and doctors -have divergent views on what is stressful in an NICU? Are events or situations stressful because of the intensity of stress they arouse, the frequency with which they occur, or both?

\section{Subjects and method}

The participants in the study were 22 paediatric consultants from 17 NICUs around Australia, and 29 NICU nursing sisters from the Queen Victoria Medical Centre. Twenty of the 22 consultants were 
male, whereas all the nursing sisters were female. Their average time spent working in an NICU was 56 months. Fourteen consultants were working full-time as neonatologists in the NICU and 8 were working part-time in neonatal paediatrics.

Ten situations previously reported as stressful ${ }^{1}$ were adapted for use in a questionnaire which respondents rated on a 4-point scale, from least to most stressful, after reading expanded verbatim quotes related to each of these areas of stress. Situations were judged for (1) the intensity of stress they aroused and (2) the frequency with which they occurred. The use of this 10-item questionnaire allowed paediatric consultants' and nursing sisters' responses to be compared on individual items, as well as their mean scores for overall intensity and frequency of stress to be calculated. Statistical tests for differences between paediatric consultants and nursing sisters were carried out using $t$ test and $\chi^{2}$ test as appropriate.

The 10 items rated for intensity and frequency of stress were presented in the following order. They were: (1) ambivalence to parents, (2) priorities of care, (3) doctor/nurse conflict (nurse/doctor conflict), (4) understaffing/overwork, (5) doctor/doctor (nurse/ nurse) problems, (6) sudden death/relapse of an infant, (7) insecurity regarding competence and knowledge, (8) impact/shock of sights and smells, (9) personal life versus work, and (10) condition of outborn infants brought in.

In addition to filling in the questionnaire which was sent out to the participants, they were asked to provide 3 recent accounts of stress which they had personally experienced while working in an NICU. Thus the individual's experience of stress, as well as the group of which he or she was a member, could be taken into account. Furthermore, any important omissions in the questionnaire would be revealed by the individual reports of stress.

\section{Results}

The rank order of items when rated for intensity of stress for paediatric consultants and nursing sisters is shown in Table 1. The percentages refer to the proportion of subjects rating items as moderately or highly stressful. The rank order of items when rated for frequency of stress is shown in Table 2. The percentages refer to the proportion of subjects rating items as recurring often or almost continuously.

Differences between paediatric consultants and nursing sisters. For intensity of stress, only one of the 10 comparisons was statistically significant. Paediatric consultants perceived the competing demands of
Table 1 Rank order of items for intensity of stress

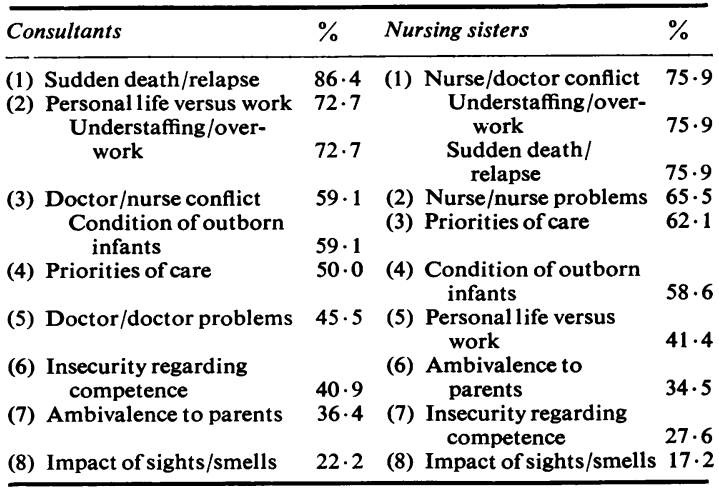

Table 2 Rank order of items for frequency of stress

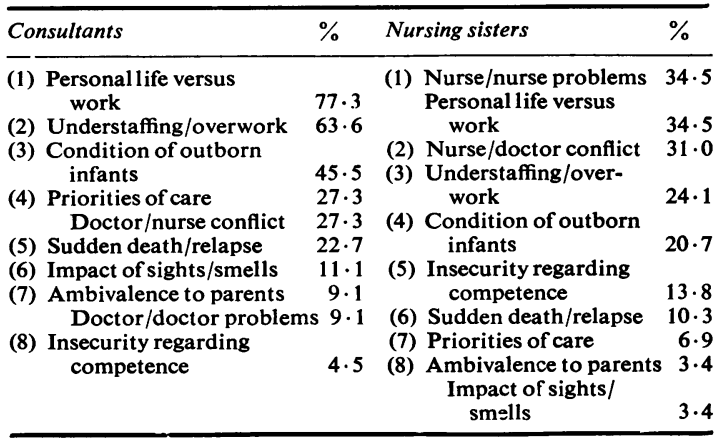

personal life and work to be more stressful than did nursing sisters $\left(\chi^{2}=4.96\right.$ with $\left.1 \mathrm{df}, P=0.02\right)$.

For frequency of stress, 5 comparisons were statistically significant. For only one of these comparisons did nursing sisters rate frequency of stress more highly than paediatric consultants. The former reported problems with their peers more frequently than the latter $\left(\chi^{2}=4.48\right.$ with $\left.1 \mathrm{df}, P=0.03\right)$. The 4 items paediatric consultants rated as more frequently stressful than nursing sisters were priorities of care $\left(\chi^{2}=3.9\right.$ with $\left.1 \mathrm{df}, P=0.04\right)$, understaffing/overwork $\left(\chi^{2}=8.05\right.$ with $1 \mathrm{df}, \mathrm{P}=$ $0 \cdot 004)$, personal life versus work $\left(\chi^{2}=9 \cdot 19\right.$ with $1 \mathrm{df}, \mathrm{P}=0.002$ ), and condition of outborn infants $\left(\chi^{2}=3.56\right.$ with $\left.1 \mathrm{df}, P=0.05\right)$.

Mean scores for intensity and frequency of stress for paediatric consultants and nursing sisters showed that there was no significant difference between the two groups for intensity of stress. However, there was a highly significant difference in their mean scores for frequency of stress (paediatric consultants 
$22 \cdot 04 \pm 3 \cdot 34$ (mean $\pm \mathrm{SD}$ ), nursing sisters $19 \cdot 13 \pm$ $3 \cdot 83, t=2 \cdot 89$, df $=47, \mathrm{P}=0 \cdot 006$ ).

Individual reports. Reports from individual paediatric consultants and nursing sisters confirmed the findings of the questionnaire by emphasising the stress associated with understaffing/overwork, nurse/ doctor conflict, the sudden death/relapse of an infant, and the condition of outborn infants brought in. From these individual reports the nurse/doctor conflict which dominated the nursing sisters' stresses appeared to be between nurses and resident or registrar staff rather than paediatric consultants. However, additional sources of stress were also identified. Paediatric consultants and nursing sisters both commented on the stress associated with (1) trying to support the parents of and care for a child with an alternating prognosis, (2) the ethical problems related to the appropriate length or intensity of treatment, (3) avoidable trauma to the infant, and (4) administrative problems. Consultants also commented on the difficulty of deciding the limits of their responsibility in the nursery.

Four paediatric consultants and 3 nursing sisters described having difficulty coping with the alternating prognosis of acute deterioration and unexpected recovery of an infant. Individual reports covered the following situations: an infant who was born alive when parents had been told he would be stillborn; telling parents that an infant would not survive the night but who was still alive the next morning; and an infant who showed a sudden improvement in his condition against all expectations. They found that parents became hostile towards them if reports and predictions about the infant's outcome vacillate, because they could not give parents the definite, once-and-for-all prognosis they hoped to hear.

Four paediatric consultants and 3 nursing sisters reported the constant stress related to the appropriate amount and length of treatment-terminable and interminable. This was summed up by one paediatric consultant who wrote of the conflict over withdrawing or withholding life support mechanisms when dealing with acute or chronic situations. He asked, 'Does the patient have a right to die versus a right to live?'

Another area of stress shared by paediatric consultants and nursing sisters related to what they saw as avoidable trauma to the infant. Two paediatric consultants and 2 nursing sisters gave relevant examples. A salient feature of this stress was the feeling of impotence and anger preventable iatrogenic problems provoked.

Five paediatric consultants and 2 nursing sisters described stress that they attributed to admini- strative decisions which directly affected the quality of care in the NICU, but which were made by those lacking understanding of its working environment and patient care requirements.

A further source of stress mentioned by 4 paediatric consultants but none of the nursing sisters concerned the limits of their responsibility. There was an anxiety over loyalty to their paediatric registrars (especially if their competence was in doubt) and to peers in another speciality, such as obstetrics, when this loyalty was in conflict with what was perceived as the best interests of an infant and his family. Finally, 3 paediatric consultants described the stress of trying to combine service, administrative, and teaching commitments with worthwhile clinical auditing and research.

\section{Discussion}

The findings of the present study into the stress associated with working in an NICU, help to clarify the relative importance of various types of stress in terms of their intensity, their frequency, and their differential impact on paediatric consultants and nursing sisters.

The results reveal the existence of a very definite hierarchy among the 10 events or situations rated for intensity of stress. At the top of the hierarchy, with more than $70 \%$ of the total sample rating themo as highly stressful, were the problems of understaffing/overwork and the sudden death/relapse of an infant. Close in importance to these were nurse/ doctor conflict, priorities of care, and the condition of outborn infants. At the other end of the hierarchy, with $40 \%$ or less of the sample finding them as highly stressful, were ambivalence to parents, insecurity regarding competence/knowledge and, least of all, the impact of sights/smells which only $22 \%$ of the paediatric consultants and $17 \%$ of the nursing sisters rated as highly stressful. The large degree of agreement between paediatric consultants' and nursing sisters' ratings was also shown by the fact that their ratings differed significantly on only one item. Paediatric consultants found more stress accommodating the dual demands of personal life and work. Significant by its unimportance both for paediatric consultants and nursing sisters is the stress related to ambivalence or conflict with parents. This suggests that the increased parent/staff contact made possible by open visiting policies is not a major contributor to stress in the NICU.

For frequency of stress, there were 5 significant differences in the ratings for paediatric consultants and nursing sisters. One of these, the conflicting demands of personal life and work, headed the list 
of items rated for frequency of stress by paediatric consultants. This stress therefore operates at both an acute and chronic level for paediatric consultants. This might be related to the fact that on-call periods for paediatric consultants could range from a weekend to up to a week, with often frequent recalls back to the NICU, in contrast to the regular shift work to which nursing sisters are rostered. The 3 other items paediatric consultants rated more highly than nursing sisters for their frequency-understaffing/ overwork, priorities of care, and condition of outborn infants-all interact and multiply overall stressfulness. The greater frequency with which paediatric consultants experienced stress was also revealed by their significantly higher mean scores on the 10 items measured. In fact, nursing sisters rated only one item, that of finding problems with their peers, as significantly more frequent in its occurrence than paediatric consultants. However, this finding may only indicate that nursing sisters, by virtue of their greater numbers in the NICU, do have peers, while paediatric consultants do not or have fewer to relate to.

Several additional sources of stress were revealed by the individual reports. While each area of concern outlined in these reports produced stress, this stress could result from quite different forms of psychological response. Thus, with unexpected recovery of an infant; the stress was derived from feelings of shock or even embarrassment, while the stress related to the morality of prolonged treatment was dependent on feelings of emotional and intellectual ambivalence, and the stress felt about avoidable trauma in an infant was mainly characterised by straightforward anger. Clearly then, while stress is a common end point, its contributing elements can range over a broad psychological spectrum.

The only central theme in these reports of stress and one which also illuminates the questionnaire findings, is that of control and predictability on the one hand and lack of control and unpredictability on the other. Thus, the physical environment of the NICU, which is a predictable given in the working lives of staff, is perceived by them as the least stressful item on the questionnaire probably because it is constant and is therefore amenable to desensitisation. However, events characterised by their unpredictability, such as sudden death or unexpected recovery of an infant, inevitably arouse stress in staff who cannot predict such events and can never therefore, emotionally inoculate themselves against them. Paediatric consultants can deal with the problem of 'burnout' by concerning themselves with less urgent paediatrics and delegating to other doctors-for example, to senior registrars. There is nothing equivalent for NICU nursing sisters who experience a higher turnover rate. Finally, there are situations over which staff feel they have no control and which consequently arouse stress derived from feelings of impotence and powerlessness but which are in reality amenable to change. Most important here is the dual conflict of understaffing/overwork and associated administrative problems. It is to these joint problems that programmes designed to reduce stress in an NICU could most profitably be directed as many apparently personal and psychological difficulties mentioned by staff stemmed directly from the onerous demands of their working conditions.

\section{References}

1 Jacobson S. Stressful situations for neonatal intensive care nurses. Am J Mat Child Nurs 1978; 3: 144-50.

2 Hay D, Oken D. The psychological stresses of intensive care unit nursing. Psychosom Med 1972; 34: 109-18.

3 Duff R S, Campbell A G M. Moral and ethical dilemmas in the special care nursery. $N$ Engl J Med 1973; 289: $890-4$.

4 Frader J E. Difficulties in providing intensive care. Pediatrics 1979; 64: 10-6.

5 Marshall R E, Kasman C. Burnout in the neonatal intensive care unit. Pediatrics 1980; 65: 1161-5.

6 Consolvo C A. Nurse turnover in the newborn intensive care unit. JOGN Nurs 1979; 8: 201-4.

Correspondence to Dr V Y H Yu, Department of Paediatrics, Queen Victoria Medical Centre, 172 Lonsdale Street, Melbourne, Victoria 3000, Australia.

Received 6 July 1981 